\title{
Ergonomic evaluation of the environment: a case study in a control room of the hydroelectric sector
}

\author{
Christianne Soares Falcão ${ }^{\mathrm{a}}$ and Marcelo Marcio Soares ${ }^{\mathrm{b}}$ \\ ${ }^{a}$ Postgraduate Program in Design of Federal University of Pernambuco, Brasil \\ ${ }^{\mathrm{b}}$ Postgraduate Program in Design of Federal University of Pernambuco, Brasil
}

\begin{abstract}
Representative systematic evaluation studies of the workspace and the extent to which that space is suitable for performing tasks have been developed by professionals engaged on finding evidence as to the importance of users and designers being joint participants in drawing up projects. In this context, this paper sets out to evaluate the environment of a control room in the hydroelectric sector, based on a multidisciplinary method which integrates ergonomics, architecture and environmental psychology so as to assess the influence of space on the user, and thus to identify the user's level of satisfaction with it. It was observed that some adaptation strategies of the space for activities were not implemented satisfactorily, resulting in the need for further studies on making workspaces suitable.
\end{abstract}

Keywords: control room, environmental ergonomics, constellation of attributes

\section{Introduction}

Identifying the suitability of an environment to the user and the tasks undertaken involves many variables, resulting in the task of measuring such suitability, which is enormously complex, especially when viewed from the standpoint of ergonomics. The side of ergonomics that is dedicated to the study of the physical environment is environmental ergonomics or ergonomics of the built environment. The methods of analysis of this segment are based on understanding the activities carried out in real work situations, considering the context and the diversity of the individual participants.

However, Parsons [4], emphasizes his concern that many ergonomic studies tend only to consider the environment in a mechanistic way, by tackling only levels of illumination and noise, for example. This conduct has not contributed to good performance in planning workplaces that are appropriate to their function and to those who use them.

The use of norms is not sufficient to guarantee good performance in designing environments. These should be used in conjunction with knowledge of the activities and demands of the work, taking into account that many of the key issues that define layout and ambiance are derived from the feelings and perception of the user.

This article presents the results of an ergonomic analysis of the built environment in a Control Room, responsible for transmitting electric energy in Northeast Brazil. Control room environments are complex and still largely unexplored environments, where the physical arrangement of the environment influences the operators' behavior, thus determining the success or otherwise of its architectonic design. The choice of location was prompted because the Control Room had undergone several phases of renovation so as to be adapted for the information system, which replaced the synoptic tables. The activities undertaken aim to keep the information system operating, by conducting command activities, maneuvers and monitoring using the monitors and screens that form a video wall.

\footnotetext{
* Corresponding author. christiannevas@hotmail.com marce-
} 1o2@nlink.com.br 
To conduct the research, an interdisciplinary approach was used, integrating Ergonomics, Architecture and Environmental Psychology, in the evaluation of the influence of space on the user. Architecture focuses on the physical environment and its relationship to human life, by adapting the environment to the users' lifestyles. Environmental Psychology seeks to discover what symbolic values of the physical space are important and Ergonomics puts human beings as the guiding element, by studying how the space is used in order to tailor it to the tasks and activities that will be performed in it.

\section{Method}

The method applied was based on the Ergonomic Method of Evaluating the Built Environment (MEBE), proposed by Villarouco [6]. According to her, the method seeks to establish an analogy between the phases of the traditional analysis and those needed to evaluate the space as the focus on the work performed in it, by verifying possible interactions that are harmful to productivity or that could lead to improved working conditions. This method consists of three steps: Overall Analysis of the Environment, Identification of the Environmental Configuration, Assessment of the Environment in Use in the performance of activities. The first phase includes analyzing the physical environmental conditions. Thereafter, a research phase begins on users' perception of the space they use based on applying Environmental Psychology tools. The assessment concludes with the diagnosis of the situation studied.

The field research included taking measurements of the physical environment and collecting data on equipment and jobs, through direct observation, interviews and questionnaires. We tried to get as much information as possible on the environment of the control room, because, among the environments of all the control center environments, it is the one which is the most complex.

The tools used in research are presented below, the order of presentation of which is the same as that for methodology: (1) Overall Analysis of the Environment (Walkthrough Analysis and Questionnaire on the users' opinion), (2) Identifying the Environmental Configuration (Surveys of physical aspects, furnishings and equipment) (3) Assessing the Environment in Use when Activities are being performed (systematic observations), (4) users' perception of the space (Questionnaire on users' opinion about the comfort of the environment and the constellation of attributes) and (5) an Ergonomic Diagnostic of the Environment.

\section{Overall analysis of the environment}

The Analysis of the Global Environment step is the first contact with the environment in which what should be sought are an understanding of the space, the work performed in and charactering the main problems. During this step, it is sought to raise as much information as possible about the environment of the control room by using Walkthrough Analysis and a Questionnaire on the Users' Opinions.

Before evaluating how the operators respond to the environmental issues of the room, there is the need to gather good knowledge of the location and social context in which the operators are inserted from the researcher's particular point of view. The Walkthrough Analysis was undertaken by making use of photographic records and notes in the field diary and the floor plan of the building. The application of this tool allowed us the positive and negative points as to the physical aspects of the environment to be identified, as well as enabling the researcher to gain greater familiarity of the space and its users, this being an important source of data for planning other research procedures adopted in the subsequent steps.

On entering the room, the first observation was that it was clean and organized. The light colors and textures of the furnishings create a pleasant environment. A negative impact is caused by the difference in materials and unevenness of the floor. After further analysis, some elements were identified as contributing to visual pollution in the room, because their placement had been improvised and was inappropriate. These were: a trolley for a television and two vases with artificial plants.

The operators work under a shift system and therefore the job is shared between them all. Thus, personal items, such as photos, potted plants and decorative items cannot be used to add personal touches that would capture the character of each of them. It was observed that in an attempt to give an identity to their place of work, the operators use personalised wallpaper on the screen of one of the monitors to prepare reports and documents. It was observed that operators value interpersonal relationships and the atmosphere between them is very relaxed.

The user opinion survey was designed for the purpose of obtaining the operators' personal and functional data and information about their job. The ob- 
jective of the questions in the questionnaire was to address various topics, which were divided into the following three main groups: Personal Data, Functional Data and Data on the workplace/ work environment.

The results obtained from the first two sets of questions show that operators belong to an adult age group (over 30 years old) and have spent a reasonable amount of time working in the company. However, what is important is that most were in the company from the time when refurbishment brought about changes to the room to when the computerized system was set up.

For the third group of questions, the main problems of the working environment highlighted by the operators were:

- Operations Desk - was appointed the operators pointed out that because the consoles are too far apart from each, they make communication difficult and how they are positioned does not allow there to be a good view of the video wall.

- Equipment fittings - the supply of power by electrical cables and structured cabling is loose, apparent, underneath the operators' consoles and was signalled as a nuisance factor. Also cited was the lack of power.

- False floor - between the video wall and the consoles, there is an area of false floor covered by metal plates that was previously used for the passage of synoptic panel cabling. The operators mentioned that because the plates are not securely fastened, they generate noises when stepped on, and moreover is a likely place in which small animals and insects find shelter.

- Noise - the noise from equipment was mentioned, especially air conditioning, it is a nuisance factor in the room, and also mentioned was the lack of insulation for other rooms.

- Furnishings and equipment - these received a smaller number (between 1 and 2) of mentions. Chairs were said to be uncomfortable, monitors poorly distributed, the phone positioned incorrectly and access to the advice folders difficult.

The main qualities of the control room pointed out were: climatization (5), comfortable space (3), good lighting (2), team harmony (2) and resources sufficient to undertake the tasks (2).

\section{Identification of the environmental configuration}

This stage dealt with assessing the environment. In it, all the physical environmental conditioners were identified ranging from the survey of the dimensions of the space, lighting, ventilation, noise, temperature, flows, layout, displacements, cladding materials and accessibility conditions. This led to the first hypotheses on the question of the influences of the space on the conduct of work activities.

The control room has three workstations for system operators, a supervisor workstation, two mobile filing cabinets with folders for consulting data and a video wall panel, which was set up recently and is still in the adaptation phase (See figure 1).

The benches of operators 1 and 2 are arranged in a central position in the room, in front of the video wall panel and house the equipment needed to perform the control activity. That of the third operator is located outside the area in which the video wall panel can be seen. The supervisor's workstation, which is still improvised as a consequence of bringing in another operator, is located opposite Operator 3.

On the bench of the workstations there is the following equipment: three 21 " monitors that display the system, a 21" monitor for communication operations with the local network and filling in report forms, a 15 "monitor for the system, a support system for alarms, a set of mouse and keyboard for each system, a printer, a pigeon-hole in-tray for reports, a PABX telephone, two spare telephones and another just for internal communication. The desk has a useful depth of $57 \mathrm{~cm}$, is U-shaped and consists of regular modules. The drawer for the keyboard is not used and is on the desk, thus reducing the usable area of the working surface, which makes the handling and filling in of documents difficult. Beside the bench there is a module with drawers for keeping personal items and office supplies in.

\subsection{Evaluation of the lighting}

Measurements of the illuminance of the room were carried out under the method of direct reading, using a digital light meter, at two times of day, in the morning (9:40) and in the evening (19:00). Points in the main points of activities in the room were marked, at the peak of the work plans. By performing a general analysis of all the points, it is observed that the illuminance levels are within the standards recommended by ISO 11064-3 [2], but are not distributed evenly. 
It was also noted that the lamps located near the video wall are always turned off to prevent glare on the screens.

\subsection{Evaluation of the acoustics}

The analysis was based on ascertaining the acoustic conditions of the environment, by noting the sources of internal noise and whether they were compatible with ISO 11064-6 [3], which recommends that the value of $45 \mathrm{~dB}$ is not exceeded in control rooms. The measurements were taken using two scenarios, one with air conditioning units turned on and the other with them turned off.

In analyzing the data obtained, to begin with it was assessed that the evaporator units of the air conditioners of the Split System type were a major source of noise, and the operators complained about this.
When taking measurements with the units turned off, although we noted a certain drop in noise in the room, the values were still higher than recommended. Therefore, it was found that when the air conditioners are turned off, the noise in the room is caused by the equipment on the video wall.

\subsection{Evaluation of the temperature}

The control room has three air conditioners of the Split System type that are kept on 24 hours a day. As there is a remote control for them, the operators are able to regulate the temperature and fan speed in accordance with their needs. They are satisfied with having this ability, and reported that many of them keep the temperature at the minimum, especially during the graveyard shift.

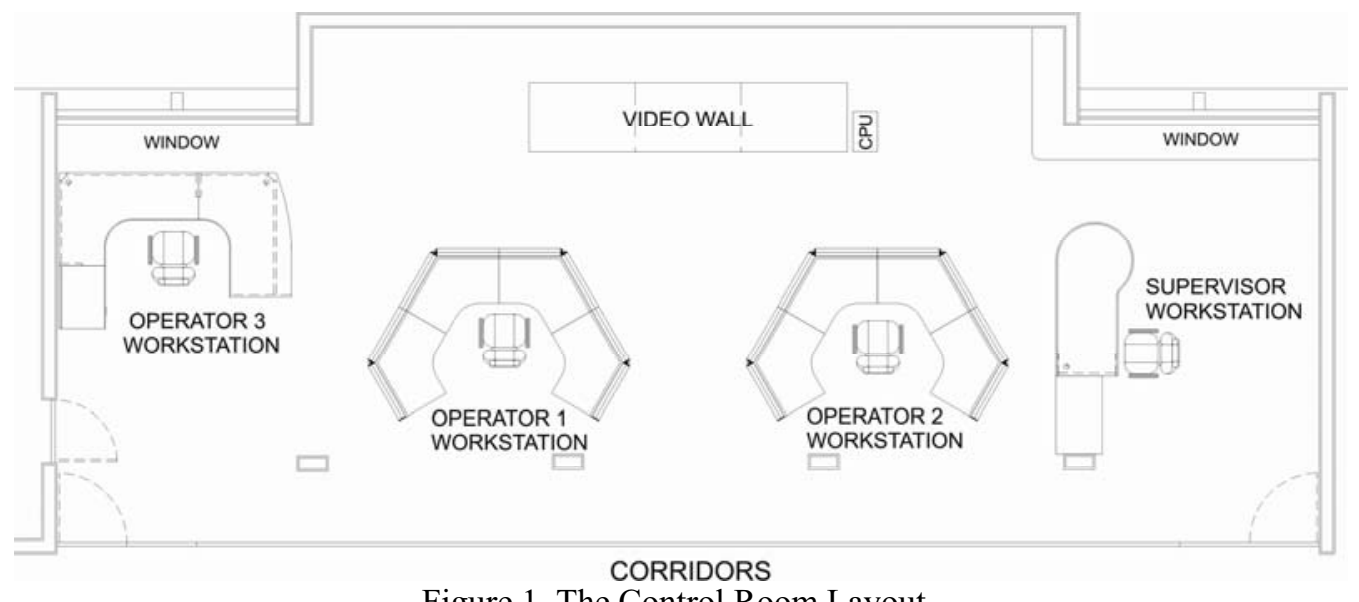

Figure 1. The Control Room Layout

\section{Assessment of the environment in use in the performance of activities}

The objective of the step of evaluating the environment in use is identify the function of the space as a facilitating element or one that hinders the performance of activities. Based on observing the operators when they were carrying out their tasks, what were noted were inadequacies in the furnishings and equipment, interferences of flows, examples of discomfort, and so on.

Operators communicate with each other constantly, and move about between workstations. However, very often, because they are on the phone or even operating the system, it is not possible for them to leave their desk to talk to a colleague. This situation often forces them to speak loudly, which disturbs the others who are focused on their activities. Operator 3, because he is in charge of the records, is always in contact with Operator 2 and with the supervisor, who are at opposite ends of the room.

The location of the three system monitors is on the bench on the left side of Operator 1, forcing him to turn his body to view them and use the mouse. Another issue observed as to the placement of the monitors is that they block the view of some parts of the video wall (See Figure 2). 


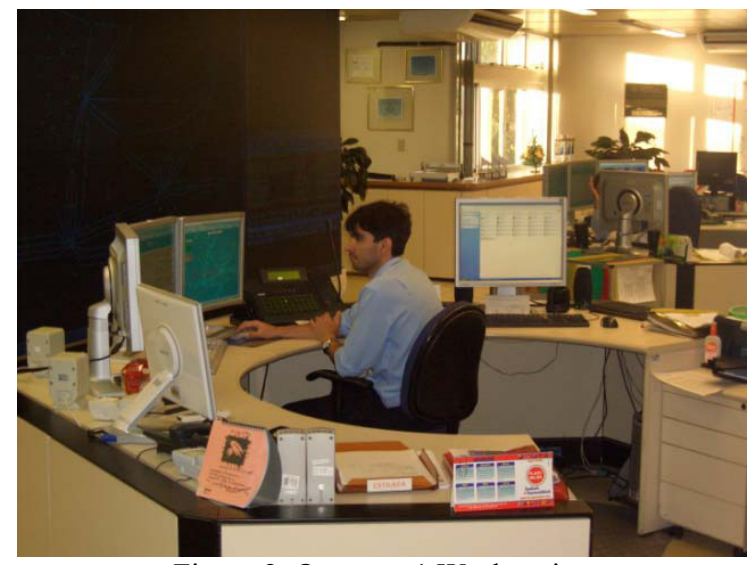

Figure 2. Operator 1 Workstation

Consulting the folders in the mobile file very often occurs while the operator is on the phone. Therefore, so as not to interrupt the connection, he leans across the table to reach them or walks over to them with the phone set resting on his shoulders and looks up the file while standing.

As the room environment has been undergoing constant modifications so as to adapt it to the computer system, some items have been left unfinished, which gives an image of improvisation and untidiness, such as uncovered electrical cables and data, the grills of the old central air conditioning system which remain in partitions, false floor boards that are loose, including many that are damaged, as well as the presence of bumps and differences in the finishing of the floor.

\subsection{Analysis of the flows}

During several visits to the control room, observations were made of the behavior of operators in their work activities. These showed that both the operators and the supervisor did not behave in a static manner, they had meetings with each other in accordance with the progress of events. Moreover, their activities were not restricted to the boundaries the Operations Desk via computerized equipment. Such activities included consulting Document folders, located in the mobile filing cabinets.

Data were acquired using Link Analysis which according to Wood [7] is a technique used to determine the communication between different team members. The observations were made both in the periods considered quiet, but also in the busiest ones.

On carrying out a general analysis of the data flows, intense communication between Operators 1 and 2 was noted, as was that of Operator 3 with Operator 2 and with the supervisor. Thus, the location of Operator 3 is far away from the points where he goes to most. The positioning of the supervisor makes it difficult to communicate with other operators, especially with Operator 3, but also with the Preoperations team. Information obtained from the analysis of the flows is of fundamental importance for the positioning of the workstations in the physical arrangement of the room. In this case, the position of the operators at separate islands, as well as the arrangement of benches in the room, cause problems in communication between them (See Figure 3).

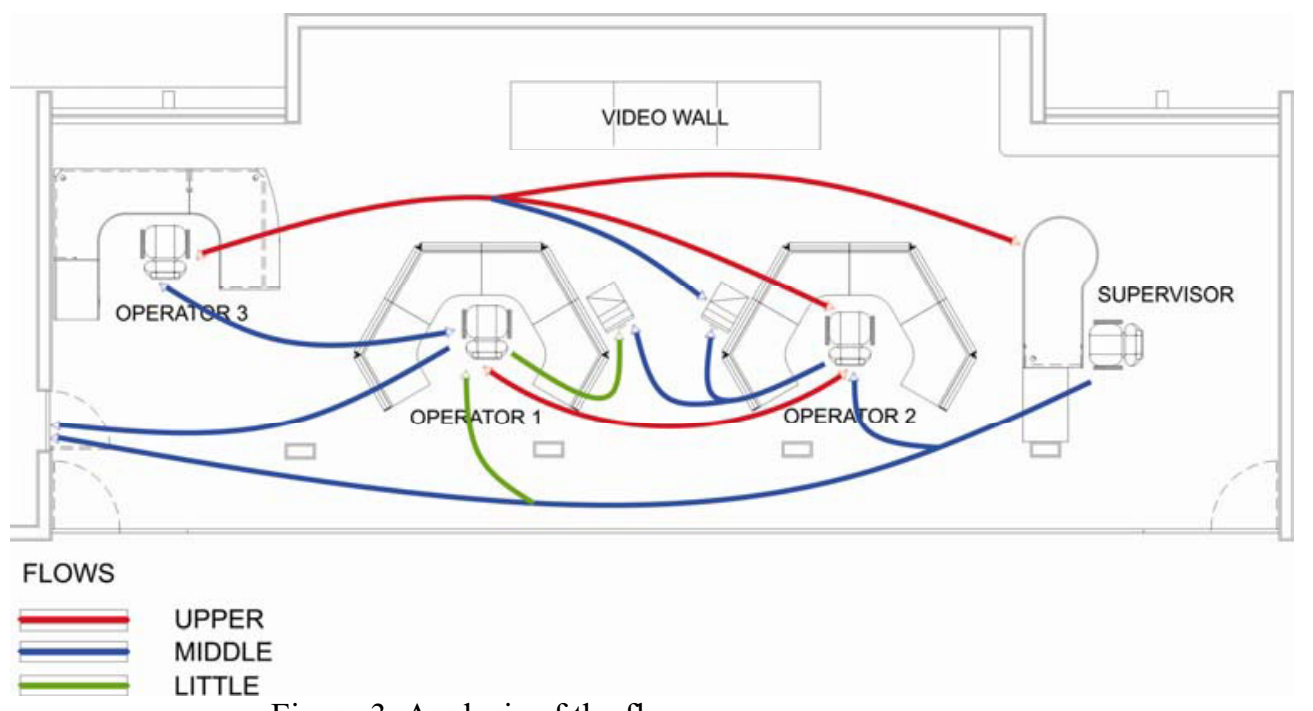

Figure 3. Analysis of the flows 


\section{Users' perception of the space}

The step of user's perception can be considered essential to evaluating the space, because it puts man as the central character of all the actions. Two tools were used in this step: a user opinion survey on the comfort of environment and the Constellation of Attributes.

According to Parsons [4], subjective evaluation is recommended for the analysis of psychological factors, such as, for example, the situation of comfort. Thus the opinion survey on the comfort of the environment was applied with users, in order to grasp their perception as to the thermal, lighting and acoustic conditions of their work environment.

As to the thermal conditions, the results showed the operators were satisfied as to the room temperature, where the vast majority chose the option that suggested the room temperature as it is.

Regarding the lighting comfort, the responses indicated that a small majority noted problems regarding the quality of lighting in the room, of whom six respondents $(46 \%)$ indicated that the lighting is comfortable. When the operators were asked for a more specific answer to the type of nuisance from lighting, this enabled the following considerations to be made:

- Video wall - The glare caused by the lamps shining on the monitors do not permit the data to be seen from some points. At some angles, especially opposite the bench, the view of the data is blurred. The excess lighting caused by sunlight coming through the window also causes glare on the panel.

- Lamps - Because of problems from defects, or perhaps lack of ongoing maintenance, the lamps flash from time to time. The lamps should be appropriately distributed in line with the position of the workstations.
With regard to acoustic comfort, unlike what was observed in the physical measurements, the responses indicated the evaporator units of the air conditioners as the main sources of noises (11) and only two responses indicated the equipment on the video wall.

The Constellation of Attributes tool [1] was applied to the operators to record their images and expectations about an imaginary environment of a control room, as well as real space, i.e., the control room where they work. The questions were as follows:

1. When you think of the environment of control rooms, in general, what ideas or images come to mind?

2. When you think of the environment of the real control room what ideas or images come to mind?

As per Figure 4, the psychological distance to the center of each attribute is determined in accordance with the number of responses, i.e., the more responses, the closer the attribute of the nucleus is. When analysing the constellation as to the real environment, it is noted that the factors most mentioned are related to people's access to the room, noise and the disturbing presence of mosquitoes. In the constellation as to the imaginary environment, silence and privacy are the main images. When these images are put into comparison with the real environment, it is perceived that they are not being met. As for environmental comfort, the temperature of the room was identified as a positive aspect. However, noise and inadequate lighting are disturbance factors as to the real environment of the control room. The consoles were identified as unfavorable as to their position. When this attribute is observed in the constellation referring to the imaginary environment, the need to adapt the furnishings to the new conditions of the activity of control is identified, especially after the setting up of the video wall. 

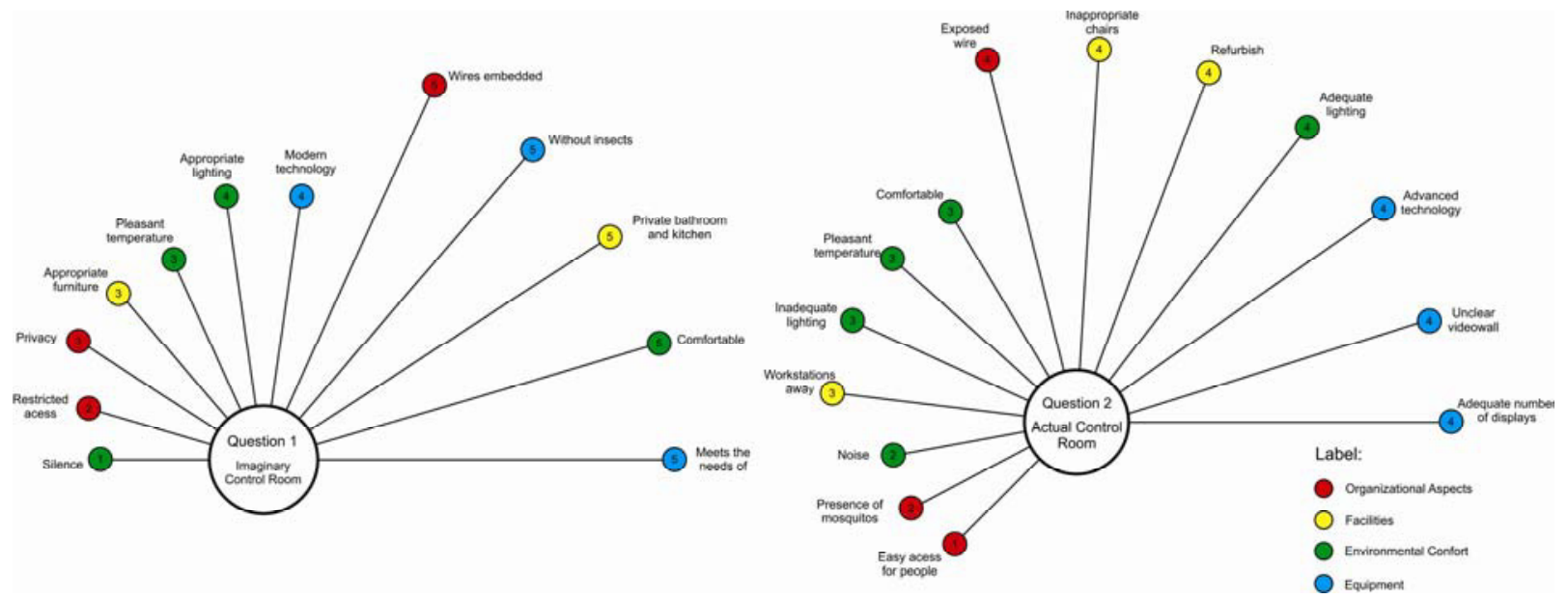

Figure 4. The Constellation of Attributes for an imaginary and the actual Control Room

\section{Ergonomic diagnostics of the environment}

In line with the data presented in the previous steps, the main environmental factors that influence the well-being and quality of the control room environment surveyed were classified, mapped as presented below.

\section{a. Physical arrangement}

In the control room, the limitations of the environment, such as structural columns, rectangular form, low headroom and access doors are factors that hinder a functional arrangement of the workstations and equipment. Despite these limitations, the panel video wall panel was set up and all the physical arrangement of the room should be rethought starting with its location [2].

The access of unwanted people was regarded as one of the main negative aspects. Because there is no environment to receive visitors, they end up coming into the room without permission. In addition to casual visits, the access to the room of employees from other sectors was pointed out in the interviews. The need for privacy is a primary factor evaluated by the operators in the Constellation of Attributes.

Despite being related to organizational aspects, the restriction on people access can be resolved from the configuration of the environment. In this case, a reception and waiting area should be set aside and separated by a glass partition from the operations area.

\section{b. Physical ambiances}

In all the tools applied, there are conflicting complaints as to the lighting. It was observed that there are places with low illumination, while in others, the lamps and natural lighting have been identified as causing glare. In this case, the need is shown for a technical study on lighting, and adding the possibility of replacing the curtains of light, usually put up in offices, with more customized systems, thus allowing the operator the flexibility to adjust the luminance according to his/her needs.

A noise-free environment is essential to control activity. It was identified that the room has important sources of noise that should be corrected. It is of paramount importance to provide a soundproof partition in the space of the video wall equipment.

\section{c. Furnishings}

The Operations Desks are important factors to be considered. The bench has been criticized because of the lack of space to house the equipment and because of its shape, thus obliging the operator to assume inadequate postures. Another issued observed is about Operator 3's bench, the position of which does not allow him to see the video wall panel. Another factor observed refers to the arrangement of separated benches which makes communication difficult. It is recommended that there is a need to group the three operators into a single cell. The supervisor's bench should be close to Operator 3's. The monitors on the bench should be distributed such that they are positioned to the front of the operators, thus avoiding their constant movement between the screens. A space for the passage of equipment wiring should be provided for on the Operations Desks. The mobile filing cabinets should be adapted to the new furnishings, and situated in the center of the cell in order to facilitate access to consulting the folders.

\section{d. Flooring}


As noted in the user opinion survey, the floor plates of the room are loose and need to be repaired, as does the inspection lid through which the cabling comes. The floor should also be leveled and covered with a single material suitable for moving the chairs.

Finally, in the analysis undertaken of the space in the control room, it was found that it is not fully appropriate for the activities undertaken, with the most recurring problems in order of highest frequency being: the need for privacy; noise; inadequate lighting; and furnishings at odds with the activity performed. Based on the recommendations proposed in this study, this gap can be filled with correct architectonic planning.

It is important to consider that besides the normal requirements of maintenance and conservation, control rooms belong to a sector that is ever evolving technology. In this sense, the changes in the environment are inherent in the evolutionary process, and spaces designed for this purpose, should as far as possible, anticipating this evolution.

The factors considered positive and contribute to the well-being and quality of the environment are: a comfortable temperature, good relationships between team members and satisfaction with the cleanliness and appearance of the physical environment.

\section{Discussion}

As a result of the experiment, from the practical ergonomic point of view allied to the architect's environmental vision, it was possible to become familiar with and experience the environment, the results of which showed the efficiency of the method applied. The analysis of the physical aspects of the environment together with the analysis of the user's perception contributed effectively to identifying the influence of the elements of comfort in performing activities. Based on a systemic approach, always focused on the user, on the elements identified as unsatisfactory and on correcting them later, this approach can promote a more suitable and satisfactory room for operators. Given the above, this paper aims to contribute to research in the area of Environmental Ergonomics, by adding another example of addressing the issues concerning the adaptation of environments to users, when performing their activities.

\section{References}

[1] Jézabelle Ekambi-Schmidt, La percepción del habitat. Barcelona: Gustavo Gili, 1974.

[2] Internacional Organization for Standartization. ISO 11064-3 Ergonomic design of control centres - Part 3: Control room layout. Genève, 2002.

[3] Internacional Organization for Standartization. ISO 11064-6 Ergonomic design of control centres - Part 6: Environmental requirements for control centres. Switzerland, 2005.

[4] Ken Parsons, The environmental ergonomics survey, In: John R. Wilson (edited by). Evaluation of human work. $3^{\mathrm{a} e d . ~ F l o r i-~}$ da: Taylor \& Francis Group, 2005.

[5] Christianne S. Falcão e Vasconcelos, Ergonomia e projetos de ambiente em salas de controle: um estudo de caso em empresa do setor hidrelétrico, Msc. Dissertation, University Federal of Pernambuco, Brasil, 2009.

[6] Vilma Villarouco, An ergonomic look at the work environment, Proceeding from IEA 09: $17^{\text {th }}$ World Congress on Ergonomics. China: Beijing, 2009.

[7] John Wood, Control Room design, In: People in Control: Human factors in control room design. Noyes, J. Bransby, M. (Ed.). United Kingdon: IEE, 2001. 